\title{
TODA EQUATIONS FOR HURWITZ NUMBERS
}

\author{
ANDREI OKOUNKOV
}

\begin{abstract}
We consider ramified coverings of $\mathbb{P}^{1}$ with arbitrary ramification type over $0, \infty \in \mathbb{P}^{1}$ and simple ramifications elsewhere and prove that the generating function for the numbers of such coverings is a $\tau$-function for the Toda lattice hierarchy of Ueno and Takasaki.
\end{abstract}

\section{Introduction}

1.1. In a recent paper [16], R. Pandharipande showed that the conjectural Toda equation for the Gromov-Witten potential $F_{\mathbb{P}^{1}}$ of the Riemann sphere $\mathbb{P}^{1}$ implies a Toda equation for the generating function of the Hurwitz numbers $H_{g, d}$. Here $H_{g, d}$ is the number of degree $d$ connected coverings of $\mathbb{P}^{1}$ by a curve of genus $g$ which have simple ramifications over some $2 g+2 d-2$ fixed points of $\mathbb{P}^{1}$. This Toda equation results in a simple recurrence relation for the numbers $H_{g, d}$.

The purpose of this paper is to prove that a more general generating function, where arbitrary ramifications are allowed over two given points of $\mathbb{P}^{1}$ (for example, over $0, \infty \in \mathbb{P}^{1}$ ) satisfies an entire hierarchy of PDE's, namely the Toda lattice hierarchy of Ueno and Takasaki [17]. This proves and generalizes the result of Pandharipande.

We will call these more general numbers of ramified coverings the double Hurwitz numbers.

1.2. Our approach is quite straightforward: we remark that the generating function for double Hurwitz numbers is almost by definition a certain matrix element in the infinite wedge space. It is a well known result of the Kyoto school that such matrix elements are $\tau$-functions of integrable hierarchies.

These Toda equations should probably be not too surprising from the physical point of view. Physicists consider the enumeration of coverings of $\mathbb{P}^{1}$ as a sort of string theory on $\mathbb{P}^{1}$, see for example [9], and relate it to a number of other models where the occurrence of integrable hierarchies is common.

1.3. It is known, see [4] and also [8, 5], that ordinary Hurwitz numbers, that is, the numbers of coverings where the ramification over $\infty \in \mathbb{P}^{1}$ is arbitrary and all other ramifications are simple, can be expressed as certain Hodge integrals over moduli spaces of curves. In this connection, it appears to be an interesting problem to:

Received May 16, 2000. 
(i) transfer the Toda equation to the Hodge integrals side,

(ii) find an expression for the double Hurwitz numbers in terms of the GW theory of $\mathbb{P}^{1}$.

1.4. The Toda lattice equations is an infinite number of PDE's satisfied by the generating function for coverings, each resulting is some recurrence relations for the double Hurwitz numbers. These relations uniquely determine the double Hurwitz numbers.

In particular, the lowest equation of the Toda hierarchy, which is the equation considered in [16] (see Sections 2.8 and 2.9 below), describes what will happen if we add an extra sheet to our covering leaving the nontrivial part of the monodromy the same.

1.5. Several recurrent relations of a different type are known in the literature, see for example [8] and references therein. It is beyond the scope of the present note to give any survey on the literature on Hurwitz numbers and their applications.

For a survey of some combinatorial and geometric aspects of Hurwitz numbers the reader is referred to [15].

1.6. I want to thank Rahul Pandharipande for helpful correspondence.

\section{Coverings and Toda equations}

2.1. Let $S(d)$ denote the symmetric group and fix some conjugacy classes

$$
C_{1}, \ldots, C_{s} \subset S(d) .
$$

Let $\operatorname{Cov}_{d}\left(C_{1}, \ldots, C_{s}\right)$ denote the weighted number of $d$-fold coverings of $\mathbb{P}^{1}$ ramified over $s$ fixed points of $\mathbb{P}^{1}$ with monodromies in the conjugacy classes $C_{1}, \ldots, C_{s}$. The weight of any covering in $\operatorname{Cov}_{d}$ is the reciprocal of the order of its group of automorphisms. This automorphisms group is the same as the centralizer of all monodromies in $S(d)$. Note that $\mathrm{Cov}_{d}$ counts all coverings, that is, including the disconnected ones.

For this weighted number there is the following formula, see for example [3], which goes, in a sense, back to Burnside (see Exercise 7 in $§ 238$ of [2])

$$
\operatorname{Cov}_{d}\left(C_{1}, \ldots, C_{s}\right)=\sum_{|\lambda|=d}\left(\frac{\operatorname{dim} \lambda}{d !}\right)^{2} \prod_{i=1}^{s} f_{C_{i}}(\lambda),
$$

where the sum is over all partitions $\lambda$ of $d, \operatorname{dim} \lambda$ is the dimension of the corresponding representation of the symmetric group $S(d)$, and

$$
f_{C_{i}}(\lambda)=\left|C_{i}\right| \frac{\chi^{\lambda}\left(C_{i}\right)}{\operatorname{dim} \lambda}
$$

Here $\left|C_{i}\right|$ is the cardinality of the conjugacy class $C_{i}$ and $\chi^{\lambda}\left(C_{i}\right)$ is the value of the irreducible character $\chi^{\lambda}$ on any element of $C_{i}$. The expression (2) is in fact a polynomial in $\lambda$ in a certain natural sense, see for example [14]. 
2.2. Recall that the Schur functions $s_{\lambda}$ are certain distinguished symmetric functions associated to any partition $\lambda$. In terms of the power-sum symmetric functions $p_{1}, p_{2}, \ldots$ they have the following expression, see [12],

$$
s_{\lambda}\left(p_{1}, p_{2}, \ldots\right)=\frac{1}{d !} \sum_{|\mu|=d} \chi^{\lambda}\left(C_{\mu}\right)\left|C_{\mu}\right| p_{\mu}
$$

where the summation is over all partitions $\mu$ of $d=|\lambda|, C_{\mu}$ is the conjugacy class corresponding to the partition $\mu$, and $p_{\mu}=\prod p_{\mu_{i}}$. We will treat the $p_{k}$ 's as independent variables.

Now let $P=\left(p_{1}, p_{2}, \ldots\right)$ and $P^{\prime}=\left(p_{1}^{\prime}, p_{2}^{\prime}, \ldots\right)$ be two sets of variables. We have the following equality:

$$
\begin{gathered}
\sum_{d=0}^{\infty} q^{d} \sum_{|\mu|=|\nu|=d} p_{\mu} p_{\nu}^{\prime} \operatorname{Cov}_{d}\left(C_{\mu}, C_{\nu}, C_{1}, \ldots, C_{s}\right)= \\
\sum_{\lambda} q^{|\lambda|} s_{\lambda}(P) s_{\lambda}\left(P^{\prime}\right) \prod_{i=1}^{s} f_{C_{i}}(\lambda),
\end{gathered}
$$

where $q$ is a new variable which keeps track of the degree $d$ of the covering. The variables $q$ is redundant since for every term its degree $d$ in $q$ is equal to both its degree in $P$ and in $P^{\prime}$, where

$$
\operatorname{deg} p_{k}=\operatorname{deg} p_{k}^{\prime}=k,
$$

but it is convenient to keep it.

2.3. Let $C_{(2)}$ be the conjugacy class of a transposition. Introduce the following generating function

$$
\tau\left(P, P^{\prime}, \beta, q\right)=\sum_{d, b, \mu, \nu} q^{d} \beta^{b} p_{\mu} p_{\nu}^{\prime} \operatorname{Cov}_{d}(C_{\mu}, C_{\nu}, \underbrace{C_{(2)}, \ldots, C_{(2)}}_{b \text { times }}) / b ! .
$$

This is the generating function for degree $d$ possibly disconnected coverings of $\mathbb{P}^{1}$ such that the ramification over $0, \infty \in \mathbb{P}^{1}$ can be fixed arbitrarily and the other $b$ ramifications are simple. It is a general property of the exponential generating functions, see e.g. Chapter 3 in [7], that

$$
\log \tau\left(P, P^{\prime}, \beta, q\right)=H\left(P, P^{\prime}, \beta, q\right),
$$

where $H\left(P, P^{\prime}, \beta, q\right)$ is the generating function for connected coverings

$$
H\left(P, P^{\prime}, \beta, q\right)=\sum_{d, b, \mu, \nu} q^{d} \beta^{b} p_{\mu} p_{\nu}^{\prime} \operatorname{Hur}_{d, b}(\mu, \nu) / b ! .
$$

Here $\operatorname{Hur}_{d, b}(\mu, \nu)$ is the weighted number of connected degree $d$ coverings of $\mathbb{P}^{1}$ with monodromy around $0, \infty \in \mathbb{P}^{1}$ being $\mu$ and $\nu$, respectively, and $b$ additional simple ramifications. The genus of such a covering is

$$
g=(b+2-\ell(\mu)-\ell(\nu)) / 2,
$$


where $\ell(\mu)$ is the number of parts of $\mu$. The weight of each covering in $\operatorname{Hur}_{d, b}(\mu, \nu)$ is the reciprocal of the order of its automorphism group. We call the numbers $\operatorname{Hur}_{d, b}(\mu, \nu)$ the double Hurwitz numbers.

We also point out that if when one of ramifications $\mu$ and $\nu$ is trivial and the covering is connected of degree $d>2$ then the automorphism group is trivial and so $H=\log \tau$ counts any such covering with weight 1 .

2.4. Denote by $f_{2}$ the polynomial (2) corresponding to the class $C_{(2)}$ of a transposition. From (3) we have

$$
\tau\left(P, P^{\prime}, \beta, q\right)=\sum_{\lambda} q^{|\lambda|} e^{\beta f_{2}(\lambda)} s_{\lambda}(P) s_{\lambda}\left(P^{\prime}\right) .
$$

Explicitly, the polynomial $f_{2}$ is given by

$$
f_{2}=\frac{1}{2} \sum_{i}\left[\left(\lambda_{i}-i+\frac{1}{2}\right)^{2}-\left(-i+\frac{1}{2}\right)^{2}\right] .
$$

Remark that the upper limit of summation can be taken to be $+\infty$ in the above sum since $\lambda_{i}=0$ for all sufficiently large $i$. Using some standard combinatorics, see for example Section 5 of [1], one can rewrite the formula (5) as follows

$$
f_{2}=\sum_{k \in \mathfrak{S}(\lambda)_{+}} \frac{k^{2}}{2}-\sum_{k \in \mathfrak{S}(\lambda)_{-}} \frac{k^{2}}{2}
$$

where

$$
\mathfrak{S}(\lambda)=\left\{\lambda_{i}-i+\frac{1}{2}\right\} \subset \mathbb{Z}+\frac{1}{2}
$$

and, by definition, for any $S \subset \mathbb{Z}+\frac{1}{2}$ we set

$$
S_{+}=S \backslash\left(\mathbb{Z}_{\leq 0}-\frac{1}{2}\right), \quad S_{-}=\left(\mathbb{Z}_{\leq 0}-\frac{1}{2}\right) \backslash S .
$$

2.5. The sum (4) admits a straightforward interpretation as a certain matrix element in the infinite wedge space. It will be convenient for us to use the notation of [13], see especially the Appendix to [13] for a summary of the infinite wedge basics. A good general reference on the infinite wedge space is Chapter 14 of [10].

Note that the power-sum variables $P$ and $P^{\prime}$ are related to the variables $t$ and $t^{\prime}$ used in [13] by

$$
t_{k}=\frac{p_{k}}{k}, \quad t_{k}^{\prime}=\frac{p_{k}^{\prime}}{k}, \quad k=1,2, \ldots,
$$

We will use the following abbreviations

$$
\Gamma_{+}=\Gamma_{+}\left(p_{1}, \frac{p_{2}}{2}, \frac{p_{3}}{3}, \ldots\right), \quad \Gamma_{-}=\Gamma_{-}\left(p_{1}^{\prime}, \frac{p_{2}^{\prime}}{2}, \frac{p_{3}^{\prime}}{3}, \ldots\right) .
$$

The formula (4) becomes

$$
\tau\left(P, P^{\prime}, \beta, q\right)=\left(\Gamma_{+} q^{H} e^{\beta F_{2}} \Gamma_{-} v_{\emptyset}, v_{\emptyset}\right),
$$


where $v_{\emptyset}$ is the vacuum vector of the infinite wedge space, $H$ is the energy operator, and $F_{2}$ is the following operator

$$
F_{2}=\sum_{k \in \mathbb{Z}+\frac{1}{2}} \frac{k^{2}}{2}: \psi_{k} \psi_{k}^{*}:,
$$

where the colons denote the normal ordering. It is clear from (6) that the operator $F_{2}$ acts as follows

$$
F_{2} v_{\lambda}=f_{2}(\lambda) v_{\lambda}
$$

where

$$
v_{\lambda}=\underline{s_{1}} \wedge \underline{s_{2}} \wedge \underline{s_{3}} \wedge \ldots, \quad\left\{s_{1}, s_{2}, \ldots\right\}=\mathfrak{S}(\lambda),
$$

is the vector corresponding to the partition $\lambda$.

2.6. Any operator of the form $\xi=\psi_{i} \psi_{j}^{*}$ satisfies the relation

$$
[\xi \otimes 1+1 \otimes \xi, \Omega]=0, \quad \Omega=\sum \psi_{k} \otimes \psi_{k}^{*},
$$

Since the operators $q^{H}$ and $e^{\beta F_{2}}$ are exponentials of sums of such operators $\xi$, we have

$$
\left[q^{H} e^{\beta F_{2}} \otimes q^{H} e^{\beta F_{2}}, \Omega\right]=0 .
$$

It follows that the sequence

$$
\tau_{n}=\left(\Gamma_{+} q^{H} e^{\beta F_{2}} \Gamma_{-} v_{n}, v_{n}\right), \quad n \in \mathbb{Z},
$$

where

$$
v_{n}=\underline{n-\frac{1}{2}} \wedge \underline{n-\frac{3}{2}} \wedge \underline{n-\frac{5}{2}} \wedge \ldots,
$$

is the vacuum vector in the charge $n$ subspace, is a sequence of $\tau$-functions for the Toda lattice hierarchy. Our old $\tau\left(P, P^{\prime}, \beta, q\right)$ is the $\tau_{0}$ term of this sequence.

2.7. We will now show that all terms of the sequence (8) can be expressed in terms of $\tau\left(P, P^{\prime}, \beta, q\right)$. We have $v_{n}=R^{n} v_{\emptyset}$, where $R$ is the translation operator, and also $\left[R, \Gamma_{ \pm}\right]=0$. We compute

$$
\begin{aligned}
R^{-n} F_{2} R^{n} & =\sum_{k>0} \frac{k^{2}}{2} \psi_{k-n} \psi_{k-n}^{*}-\sum_{k<0} \frac{k^{2}}{2} \psi_{k-n}^{*} \psi_{k-n} \\
& =\sum_{k \in \mathbb{Z}+\frac{1}{2}} \frac{(k+n)^{2}}{2}: \psi_{k} \psi_{k}^{*}:+\sum_{k=1 / 2}^{n-1 / 2} \frac{k^{2}}{2} \\
& =F_{2}+n H+\frac{n^{2}}{2} C+\frac{n\left(4 n^{2}-1\right)}{24}
\end{aligned}
$$

where $H$ and $C$ are the energy and charge operators. Similarly,

$$
R^{-n} H R^{n}=H+n C+\frac{n^{2}}{2} .
$$


Since the charge operator $C$ commutes with $\Gamma_{ \pm}$and $C v_{\emptyset}=0$, we have

$$
\begin{aligned}
\tau_{n} & =\left(\Gamma_{+} q^{H} e^{\beta F_{2}} \Gamma_{-} v_{n}, v_{n}\right) \\
& =\left(\Gamma_{+} R^{-n} q^{H} e^{\beta F_{2}} R^{n} \Gamma_{-} v_{\emptyset}, v_{\emptyset}\right) \\
& =q^{n^{2} / 2} e^{n\left(4 n^{2}-1\right) \beta / 24}\left(\Gamma_{+}\left(e^{n \beta} q\right)^{H} e^{\beta F_{2}} \Gamma_{-} v_{\emptyset}, v_{\emptyset}\right) \\
& =q^{n^{2} / 2} e^{n\left(4 n^{2}-1\right) \beta / 24} \tau\left(P, P^{\prime}, \beta, e^{n \beta} q\right) .
\end{aligned}
$$

Substituting this expression into the Hirota bilinear equations for the Toda lattice hierarchy, we obtain the following

Theorem. The function $\tau\left(P, P^{\prime}, \beta, q\right)$ satisfies the following Hirota-type bilinear equations:

$$
q^{m+1} e^{m(m+1) \beta / 2}\left[z^{-1-m}\right] e^{-2 \sum n s_{n} / z^{n}} \diamond \diamond=\left[z^{m+1}\right] e^{2 \sum n s_{n}^{\prime} / z^{n}} \boldsymbol{Q} \boldsymbol{\Lambda},
$$

where $m \in \mathbb{Z}, z$ is a formal variable, $\left[z^{m+1}\right]$ denotes the coefficient of $z^{m+1}$, $S=\left(s_{1}, s_{2}, \ldots\right)$ and $S^{\prime}=\left(s_{1}^{\prime}, \ldots\right)$ are two arbitrary sequences,

$$
\begin{aligned}
& \diamond=\tau\left(P+S, P^{\prime}+S^{\prime}+\vec{z}, \beta, e^{(m+1) \beta} q\right), \boldsymbol{\beta}=\tau\left(P+S-\vec{z}, P^{\prime}+S^{\prime}, \beta, e^{m \beta} q\right), \\
& \oslash=\tau\left(P-S, P^{\prime}-S^{\prime}-\vec{z}, \beta, e^{-\beta} q\right), \quad \boldsymbol{\phi}=\tau\left(P-S+\vec{z}, P^{\prime}-S^{\prime}, \beta, q\right),
\end{aligned}
$$

and $\vec{z}=\left(z, z^{2}, z^{3}, \ldots\right)$.

Remark that, as pointed out earlier,

$$
\begin{aligned}
\tau\left(P, P^{\prime}, \beta, q\right) & =\tau\left(q p_{1}, q^{2} p_{2}, q^{3} p_{3}, \ldots, P^{\prime}, \beta, 1\right) \\
& =\tau\left(P, q p_{1}^{\prime}, q^{2} p_{2}^{\prime}, q^{3} p_{3}^{\prime}, \ldots, \beta, 1\right) .
\end{aligned}
$$

2.8. In particular, setting $m=0$ and taking the coefficient of $s_{1}$ gives

$$
\tau \frac{\partial^{2} \tau}{\partial p_{1} \partial p_{1}^{\prime}}-\frac{\partial \tau}{\partial p_{1}} \frac{\partial \tau}{\partial p_{1}^{\prime}}=q \tau\left(\ldots, e^{\beta} q\right) \tau\left(\ldots, e^{-\beta} q\right) .
$$

This is equivalent to

$$
\frac{\partial^{2}}{\partial p_{1} \partial p_{1}^{\prime}} \log \tau=q \frac{\tau\left(\ldots, e^{\beta} q\right) \tau\left(\ldots, e^{-\beta} q\right)}{\tau^{2}} .
$$

Recall that

$$
\log \tau=H
$$

is the generating function for the connected coverings.

The combinatorial meaning of the operator $\frac{\partial^{2}}{\partial p_{1} \partial p_{1}^{\prime}}$ is that it peels off a sheet of the covering which is unramified over the special points $0, \infty \in \mathbb{P}^{1}$. The equation (10) gives thus a recursive procedure for the computation of the double Hurwitz numbers with a fixed nontrivial part of the monodromy around $0, \infty$. 
2.9. In the particular case when there is no ramification over $0, \infty$, that is, when,

$$
p_{2}=p_{3}=\cdots=p_{2}^{\prime}=p_{3}^{\prime}=\cdots=0
$$

the function $\tau$ depends on the variables $p_{1}, p_{1}^{\prime}$, and $q$ only via their product $q p_{1} p_{1}^{\prime}$. If we set

$$
u=\log q p_{1} p_{1}^{\prime}
$$

then the equation (10) becomes

$$
e^{-u} \frac{\partial^{2}}{\partial u^{2}} \log \tau=\frac{\tau(u+\beta, \beta) \tau(u-\beta, \beta)}{\tau^{2}},
$$

which is equivalent to the Toda equation derived in [16].

\section{References}

[1] S. Bloch and A. Okounkov, The character of the infinite wedge representation, Adv. Math. 149 (2000), 1-60, alg-geom/9712009.

[2] W. Burnside, Theory of groups of finite order, 2nd edition, Cambridge University Press, 1911.

[3] R. Dijkgraaf, Mirror symmetry and elliptic curves, The Moduli Space of Curves, Texel Island, 1994, pp.149-163, Progr. Math. 129 (1995), Birkhäuser.

[4] T. Ekedahl, S. Lando, M. Shapiro, and A. Vainshtein, On Hurwitz numbers and Hodge integrals, C. R. Acad. Sci. Paris Sr. I Math. 328 (1999), 1175-1180, math. AG/9902104.

[5] T. Graber and R. Vakil, Hodge integrals and Hurwitz numbers via virtual localization, math. AG/0003028.

[6] E. Getzler, The Virasoro conjecture for Gromov-Witten invariants, Algebraic geometry: Hirzebruch 70, Warsaw, 1998, 147-176, Contemp. Math. 241 (1999).

[7] I. P. Goulden and D. M. Jackson, Combinatorial enumeration, John Wiley \& Sons, 1983.

[8] I. P. Goulden, D. M. Jackson, and R. Vakil, The Gromov-Witten potential of a point, Hurwitz numbers, and Hodge integrals, math.AG/9910004.

[9] D. J. Gross and W. Taylor, Two-dimensional QCD and strings, hep-th/9311072.

[10] V. Kac, Infinite dimensional Lie algebras, Cambridge University Press, 1990.

[11] M. Kontsevich, Intersection theory on the moduli space of curves and the matrix Airy function, Comm. Math. Phys. 147 (1992), 1-23.

[12] I. G. Macdonald, Symmetric functions and Hall polynomials, Oxford Math. Monogr. (1995), Clarendon Press.

[13] A. Okounkov, Infinite wedge and random partitions, math.RT/9907127.

[14] A. Okounkov and G. Olshanski, Shifted Schur functions, Algebra i Analiz 9 (1997), 73-146 (russian); translation in St. Petersburg Math. J. 9 (1998), 239-300.

[15] A. Okounkov and R. Pandharipande, Gromov-Witten theory, Hurwitz numbers, and matrix models, in preparation.

[16] R. Pandharipande, The Toda equations and the Gromov-Witten theory of the Riemann sphere, math. AG/9912166.

[17] K. Ueno and K. Takasaki, Toda lattice hierarchy, Adv. Studies in Pure Math. 4 (1984), Group Representations and Systems of Differential Equations, 1-95.

[18] E. Witten, Two-dimensional gravity and intersection theory on moduli space, Surveys in Diff. Geom. 1 (1991), 243-310.

E-mail address: okounkov@math.berkeley.edu 